\title{
Résultats et connaissances acquises grâce à des cultures intensives de microalgues sur lisier de porcherie
}

\author{
L. Dabbadie 1
}

Mots clés : Algues, déchet organique, "aquaculture, lagunage, phytoplancton, lisier.

Pour apporter des éléments de gestion destinés à une station pilote de lagunage mise en place au cours de l'été 1992 à Château-Thierry, de nombreuses expérimentations ont été effectuées à petite échelle au Muséum national d'Histoire naturelle de Paris. Des microalgues vertes du groupe des volvocales et des chlorococcales sont utilisées pour mobiliser les nutriments contenus dans le lisier de porc. Il s'agit essentiellement de Scenedesmus falcatus Chod. et de $S$. quadricauda (Turp.) de Breb., espèces qui présentent une forte affinité pour l'azote ammoniacal, forme d'azote prépondérante dans le lisier. De très bonnes productions algales (jusqu'à $315{\mathrm{~g} \mathrm{MS} . \mathrm{m}^{-2} \text {.semaine }}^{-1}$ ) et une épuration parfois totale de certains éléments azotés comme l'azote ammoniacal ont été obtenues. Pourtant, pendant l'hiver, ces performances sont nettement moins bonnes. Un système de chauffage permettrait de remédier à ce problème, mais il est encore nécessaire d'étudier la rentabilité économique de tels dispositifs. Les proliférations de zooplancton (daphnies, rotifères) sont susceptibles de provoquer une disparition du phytoplancton en raison du broutage excessif, mais il est aisé de les contrôler en empoissonnant les cultures et en augmentant le temps de rétention de l'eau dans les enceintes.

\section{Results and knowledge obtained from intensive cultures of microalgae with pig manure}

Keywords : Algae, organic detritus, aquaculture, stabilization pond, phytoplancton, pig manure.

Many experiments have been carried on at the Muséum national d'Histoire naturelle (Paris) with 7001 tanks to improve the pilot plant built in Château-Thierry during spring 1992. Microscopic green algae belonging to Volvocaceae and Chlorococcaceae have been used for mobilizing nutrients of pig manure. The main species used, Scenedesmus falcatus Chod. and $S$. quadricauda (Turp.) de Breb., prefer environments enriched with ammonia, the most important nitrogenous part of pig manure. Algal production has often been substantial (up to $315 \mathrm{~g} \mathrm{DM} . \mathrm{m}^{-2}$. week-1) and removal of ammonia sometimes reached $100 \%$. However, during winter, production is much lower. A heating system may increase this, but it is still necessary to study the economy of the operation. Excessive zooplankton developments. (Daphnia, Rotifers) induce algal populations to collapse, but it is very easy to avoid this by introducing fishes in tanks and increasing the duration of water retention.

\section{Introduction}

De nombreux débouchés sont envisageables pour les microalgues produites de façon intensive (de la Nouë \& Proulx, 1986, de la Nouë et al., 1990, de Pauw et al., 1980), mais il semble que l'épuration d'effluents organiques tels que les déchets d'élevages soit prometteuse, car elle fournit une solution originale et relativement peu onéreuse à un problème qui devient crucial dans certaines régions (Pays-Bas,

1. Laboratoire d'Ichtyologie générale et appliquée, Muséum national d'Histoire naturelle, 43, rue Cuvier, 75231 Paris cedex 05 et Chaire de Zootechnie, E.N.S.A. Montpellier, 2; place Viala, 34030 Montpellier cedex.
Bretagne, etc...). Des recherches sont actuellement menées sur une station pilote de lagunage proche de Château-Thierry (Aisne). Sur ces installations, des microalgues sont produites sur du lisier de porc et servent à alimenter un élevage de daphnies (Daphnia magna Straus.). Ces dernières constituent un aliment de qualité pour des poissons (carpes) qui sont élevés en bout de chaîne (Combres et al., sous presse). Des expérimentations à petite échelle avaient été réalisées auparavant pour fournir des enseignements sur la gestion de ces bassins de $300 \mathrm{~m}^{2}$ et éviter des erreurs lourdes de conséquences. Des résultats partiels ont déjà été publiés (Dabbadie \& Gagnon, sous presse). Ils portaient sur différentes nuisances observées dans les cultures, et sur les 
moyens qui avaient été utilisés pour les éviter. Cet article constitue un bilan du suivi réalisé pendant une année sur ces milieux. Il porte sur les caractéristiques du phytoplancton et sur l'évolution des sels nutritifs apportés par le lisier. Sur un point particulier (les développements de zooplancton qui provoquent des effondrements des cultures), il complète les résultats présentés dans l'article précédent.

\section{Matériel et méthodes}

Les expérimentations ont été effectuées au Muséum national d'Histoire naturelle de Paris du 13 mai 1991 au 7 juillet 1992 afin de mieux comprendre la réponse d'une culture d'algues à des apports importants de nutriments (lisier de porc).

Les cultures algales ont été réalisées à l'extérieur, dans trois enceintes en plastique de 700 litres de volume utile et de $35 \mathrm{~cm}$ de profondeur. Le 17 avril 1992 , une quatrième enceinte identique $(7001,35 \mathrm{~cm}$ de profondeur) a été ajoutée pour les besoins de l'expérience.

\subsection{Mesures et prélèvements effectués dans les cul- tures d'algues}

Un suivi physico-chimique et biologique a été effectué une fois par semaine du 13 mai 1991 au 7 juillet 1992. Les prélèvements et les mesures ont eu lieu le matin, entre 9 h 30 et 11 h.

La température (en ${ }^{\circ} \mathrm{C}$ ) et la concentration en oxygène dissous (en $\mathrm{mg} . \mathrm{l}^{-1}$ ) ont été mesurées en subsurface (10 cm de profondeur environ) à l'aide d'un oxythermomètre Ponselle à lecture digitale directe. Les pourcentages de saturation ont été calculés à partir de la table de Dussart (1966). Le pH a été mesuré en subsurface avec un pHmètre Ponselle.

Un échantillon d'eau de 1,5 litres a été prélevé pour les analyses chimiques, le dosage de la chlorophylle a, et la mesure des matières en suspension. Un autre échantillon $(50 \mathrm{ml})$ a immédiatement été formolé $5 \%$ pour identification et numération des algues au microscope inversé. Le zooplancton a été récolté en filtrant 51 de la culture algale sur un filet d'une maille de $40 \mu \mathrm{m}$. Le matériel collecté a ensuite été fixé au formol à $5 \%$.

\subsection{Analyses et observations réalisées au laboratoire}

Avant d'être soumis aux analyses, les échantillons ont été filtrés sur deux filtres Whatmann GF/C $(1,2 \mu \mathrm{m})$ successivement. Le volume filtré a été de
0,251 pour chacune des deux filtrations, soit 0,51 en tout. Le poids de matières en suspension (en $\mathrm{g}$ de MES) a été estimé par la variation de poids d'un des deux filtres Whatmann GF/C avant filtration, et après filtration et dessication (à poids constant). La concentration en chlorophylle a a été estimée par mesure de la densité optique à $663 \mathrm{~nm}$ (avec un spectrophotomètre $\mathrm{HACH} \mathrm{DR} / 2000$ ), après extraction à l'acétone de la chlorophylle fixée sur le deuxième filtre Whatman $\mathrm{CF} / \mathrm{C}$ utilisé.

L'azote ammoniacal (en mg.l-1 N-NH 4 ), l'azote nitrique (en mg.l-1 $\mathrm{N}-\mathrm{NO}_{3}$ ), l'azote nitreux (en mg.l-1 $\mathrm{N}-\mathrm{NO}_{2}$ ) et l'(ortho)phosphate (en mg.1-1 $\mathrm{PO}_{4}{ }^{3-}$ ) ont été mesurés avec le spectrophotomètre HACH (DR/2000) en utilisant des réactifs de même marque et en employant les méthodes élaborées par cette compagnie (HACH 1991).

Les algues ont été identifiées au microscope inversé $(200 \mathrm{X})$ à l'aide des planches et clés d'identification de Ward \& Whipple (1918), Bourrelly $(1981,1985,1990)$ et Cardinal (1979). Le pourcentage de chaque espèce a été calculé. Des numérations ont aussi été faites selon la méthode d'Utermöhl (1958) après une décantation de 24 heures, par comptage exhaustif des algues présentes dans un sous échantillon de volume connu dilué 100 fois. Les organismes du zooplancton ont été déterminés au microscope inversé (100X) à l'aide des ouvrages de Ward \& Whipple (1918), Amoros (1984), Pourriot \& Francez (1986), puis dénombrés dans un volume connu.

Le rayonnement a été obtenu à partir des informations fournies par la Météorologie nationale (valeurs pour Paris).

\subsection{Protocole expérimental}

Le suivi des populations algales a été réalisé entre le 13 mai 1991 et le 7 juillet 1992 dans 3 puis 4 enceintes de 7001 . En début de culture, chacune a reçu un inoculum d'algues dont le volume représentait $20 \%$ du volume total. Cet inoculum provenait d'une culture algale plurispécifique, réalisée de façon permanente sur le site expérimental. Cette culture se trouvait dans les mêmes conditions que les cultures étudiées. A partir du 11 octobre 1991, deux carpes communes (poids individuels compris entre 130 et $215 \mathrm{~g}$ ) ont été mises dans chaque milieu pour évaluer leur capacité à contrôler les développements de cladocères dans les enceintes. 
Trois lisiers différents ont été utilisés. Leurs compositions et surtout leurs teneurs en matières sèches ont été très différentes (Tableau 1) en raison de la variabilité de ces caractéristiques au cours du temps au sein de l'élevage porcin d'où ils provenaient. Le lisier utilisé à partir du 26 septembre 1991 a été dilué 3 fois, en raison de sa très grande richesse en éléments minéraux et organiques. La mesure de l'azote ammoniacal a servi à déterminer le volume de fertilisant à épandre (entre 0 et 41 par jour et par enceinte). La fertilisation est apportée en même temps qu'un certain volume d'eau (de 30 à 2001 suivant la saison). Ce volume représente le flux. Le même volume de culture d'algue est retiré avant tout nouvel apport et sert à alimenter des élevages de zQoplancton (daphnies). Le temps de rétention de l'eau dans les cultures d'algues est déterminé par le rapport entre le volume de la culture et le flux journalier. Afin de favoriser le développement de Scenedesmus sp. de grande taille, le temps de rétention a été de $12 \mathrm{j}$ au cours du printemps 1992 (au printemps 1991 , il était de $3 \mathrm{j}$ ) et de $23 \mathrm{j}$ au lieu de 15 en hiver. A partir du 17 avril, la fertilisation organique a été interrompue dans deux enceintes expérimentales $\left(\varphi_{1}\right.$ et $\left.\varphi_{2}\right)$ qui ont alors reçu une fertilisation minérale composée d'ammonitrate et de superphosphate (Tableau 1), afin d'étudier l'in- fluence de ce type de fertilisation. Les deux autres enceintes $\left(\varphi_{3}\right.$ et $\left.\varphi_{4}\right)$ ont reçu du lisier de porc.

Tableau 1. Composition des différents fertilisants utilisés au cours des expérimentations.

Table 1. Chemical composition of the fertilizers used during experiments.

\begin{tabular}{ccccc}
\hline Composition & Lisier 1 & Lisier 2 & Lisier 3 & $\begin{array}{c}\text { Fertilisant } \\
\text { minéral }\end{array}$ \\
\hline $\begin{array}{c}\text { Azote ammoniacal } \\
\left(m g ~ N .1^{-1}\right)\end{array}$ & 1600 & 1030 & 3600 & 4441 \\
Nitrate (mg N.1-1) & 520 & 580 & 660 & 6000 \\
Nitrite (mg N.1-1 & 2,5 & 11,2 & 6 & 6 \\
Phosphate & 320 & 290 & 1680 & 2800 \\
$\quad\left(m g\right.$ PO $_{4}^{3-.1-1)}$ & & & & \\
Matière sèche (\%) & 0,64 & 0,52 & 12 & \\
\hline
\end{tabular}

\section{Résultats}

\subsection{Température et rayonnement}

La figure 1 présente l'évolution de la température de l'eau et du rayonnement au cours du suivi annuel. Les températures estivales ont avoisiné $20^{\circ} \mathrm{C}$, alors qu'en hiver, elles oscillaient autour de $5^{\circ} \mathrm{C}$. Le gel de la couche d'eau superficielle est apparu à trois reprises : du 9 au 16 décembre 1991, du 22 au 27 janvier 1992 et du 18 au 21 février 1992. Le rayonnement en hiver a été trois fois plus faible qu'en été ( $5000 \mathrm{~J} . \mathrm{m}^{-2}$ en hiver contre $15000 \mathrm{~J} . \mathrm{m}^{-2}$ en été).

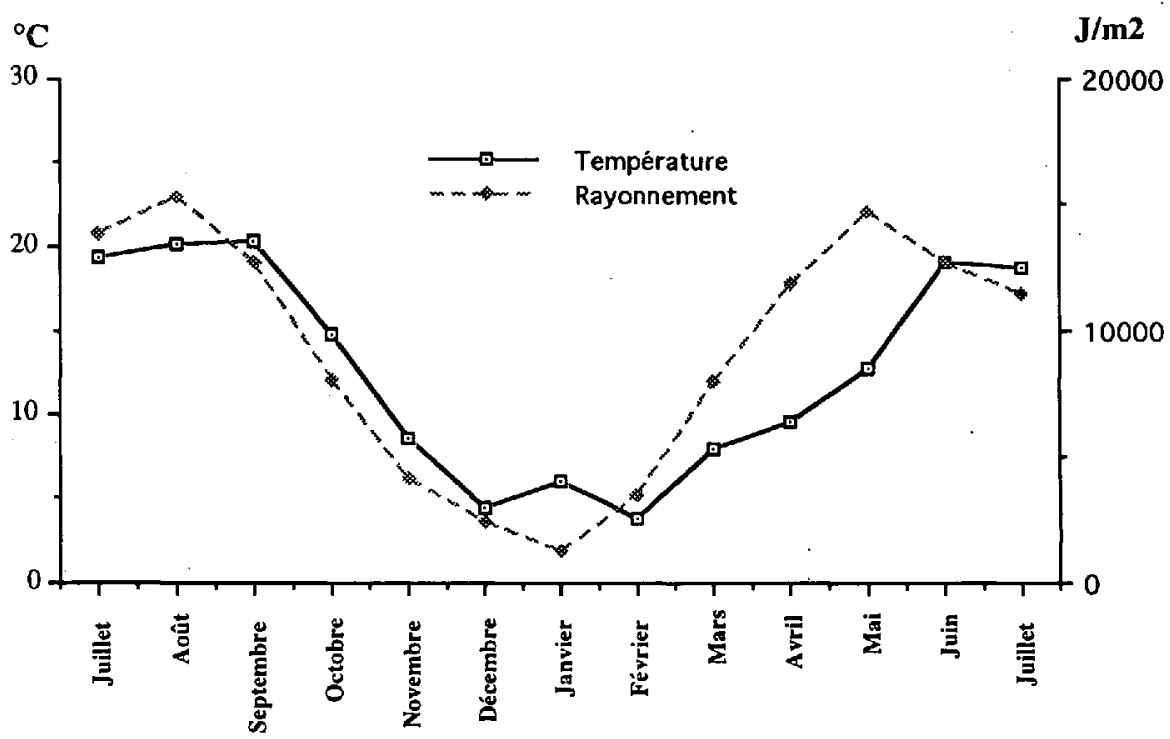

Fig. 1. Evolutions de la température de l'eau et du rayonnement perçu par les cultures.

Fig. 1. Temperature of water and solar radiation during the experiment. 


\subsection{Algues}

\section{- Systématique}

Les populations sont faiblement diversifiées. Les espèces les plus fréquentes sont des algues du genre Scenedesmus (S. falcatus Chod. et S. quadricauda (Turp.) de Breb.). D'autres apparaissent périodiquement. Elles peuvent être dominantes (volvocales et notamment Chlamydomonas sp., Cryptomonas sp., et diatomées) ou faiblement représentées (diatomées pennées et centriques, nanoplancton (algues dont la taille est inférieure à $5 \mu \mathrm{m}$ ), chlorococcales des genres Ankistrodesmus sp. ou Pediastrum sp.).

Si les populations sont parfois presque monospécifiques ou monogenres (Fig. 2), les cultures comportent le plus souvent plusieurs espèces, mais celles-ci sont toujours en nombre restreint ( 5 espèces dominantes le plus souvent). La composition spécifique de l'inoculum a eu une influence sur la composition de la culture, mais son effet a été limité dans le temps, ainsi qu'en témoignent les évolutions divergentes des enceintes $\varphi_{1}, \varphi_{2}, \varphi_{3}$ et $\varphi_{4}$ (qui ont reçu le même inoculum) après le 14 avril. Des diatomées se sont développées en masse au printemps.

Des évolutions de la composition spécifique se sont manifestées en fonction de l'état trophique des cultures. Ainsi, lorsque le milieu est devenu particulièrement riche en matières organiques après des apports excessifs de lisier, des volvocales (Chlamydomonas sp. et autres phytoflagellés) ont succédé aux chlorococcales, et lorsqu'il est devenu putride, des Cryptomonas sp. ont colonisé la culture.
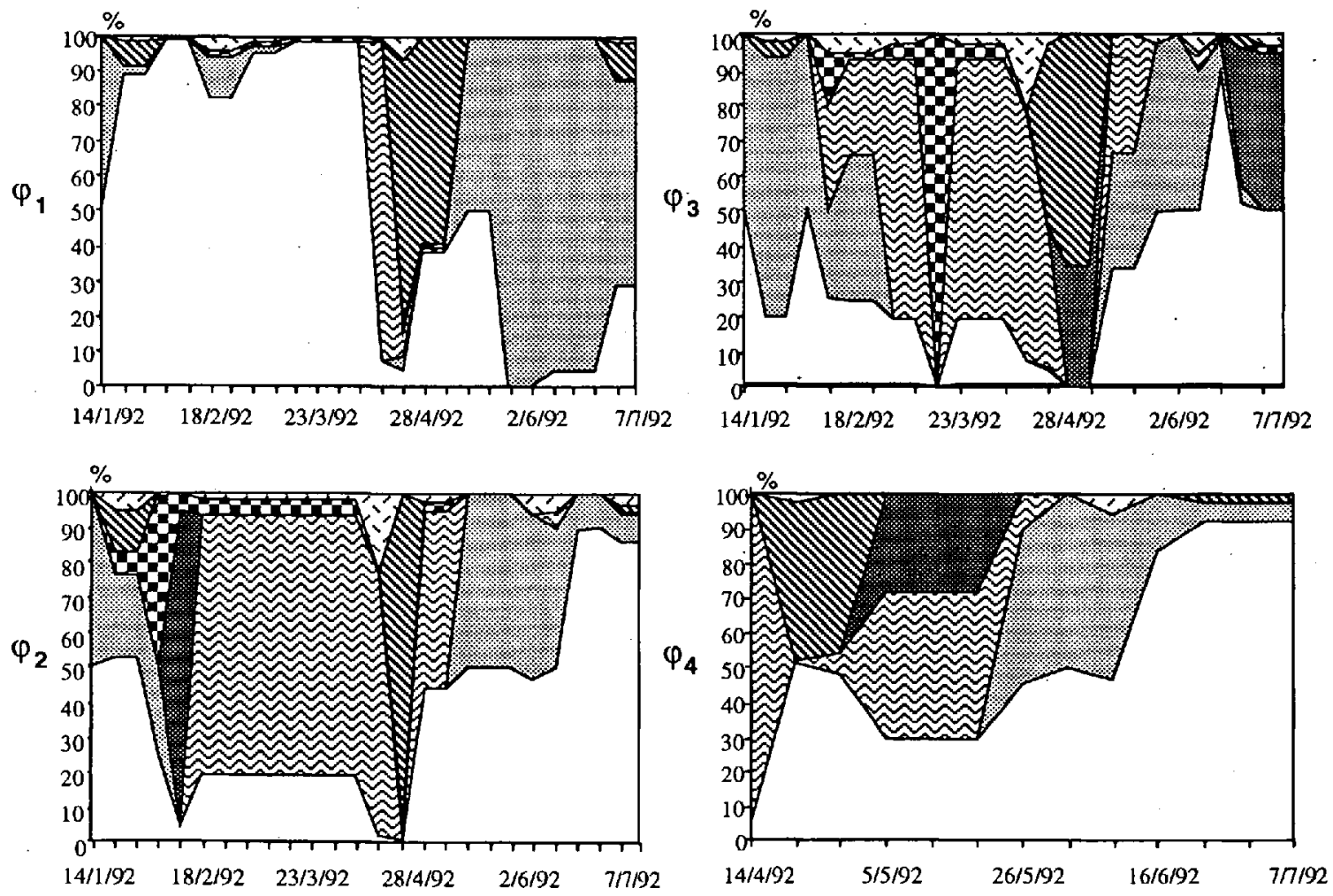

S.quadricauda S.falcatus
6 Diatomées Cryptomonas
2 Volvocales Nanoplancton

Fig. 2. Evolutions de la composition spécifique (en pourcentage de chaque espèce) dans quatre enceintes expérimentales.

Fig. 2. Specific composition (in percentage of each species) in four experimental tanks. 
Si la situation s'améliore (à la suite d'une oxygénation artificielle notamment), l'évolution se fait en sens inverse : Cryptomonas - Chlamydomonas Scenedesmus (Fig. 2, en $\varphi_{4}$ notamment).

\section{- Biomasse}

La concentration en chlorophylle a permet d'apprécier l'abondance des algues, mais la corrélation n'est pas toujours très bonne entre les valeurs mesurées et le nombre de cellules. Ainsi, le 21 avril 1992, la culture $\varphi_{2}$ contenait 250 millions de cellules par litre et sa concentration en chlorophylle a était de $145{ }_{\mu} \mathrm{G} .1^{-1}$. Le $1^{\text {er }}$ juillet 1992 , elle contenait 225 millions de cellules par litre et $297,5 \mu \mathrm{g} .1^{-1}$ de chlorophylle a.

La concentration en chlorophylle a observée pendant l'hiver a été beaucoup plus faible que pendant les autres saisons (Fig. 3). L'évolution particulière de $\varphi_{1}$ entre décembre et février montre cependant qu'il est possible d'obtenir de bonnes productions hivernales $\left(478 \mu \mathrm{g} .1^{-1}\right)$.
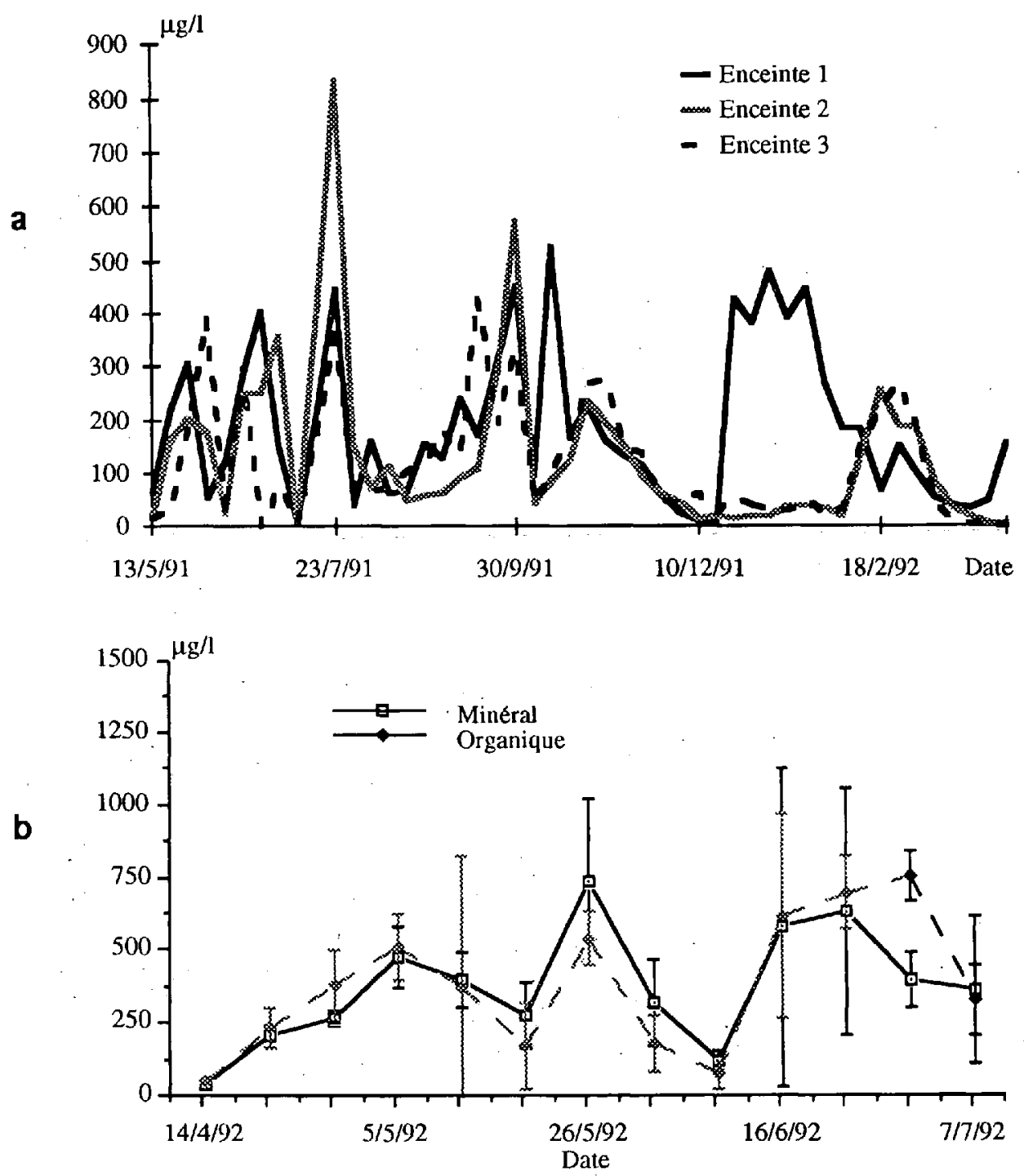

Fig. 3. Evolutions des concentrations en chlorophylle a dans trois enceintes du 13 mai 1991 au 7 avril 1992 (a) et dans quatre enceintes (b) entre le 14 avril et le 7 juillet 1992 (moyenne et valeurs pour les deux enceintes qui reçoivent le fertilisant de même nature).

Fig. 3. Chlorophyll a concentrations in three tanks from May $13^{\text {th }} 1991$ to April $7^{\text {th }} 1992$ (a) and in four tanks (b) from April $14^{\text {th }}$ to July $7^{\text {th }}$ (average and extreme values for two tanks receiving the same fertilizer). 
Les productions du printemps 1992 ont été supérieures à celles obtenues la même saison de l'année précédente et ont augmenté de façon régulière jusqu'au 16 juin 1993 où la concentration maximale a été de $935 \mu \mathrm{g} . \mathrm{I}^{-1}$ dans une enceinte fertilisée minéralement.

Les concentrations obtenues avec les cultures recevant du lisier ont été voisines, et même souvent supérieures à celles des enceintes recevant un engrais minéral (Fig. 3 b).

La teneur en chlorophylle a est caractérisée par de très brusques variations, et notamment par de fortes baisses qui suivent des explosions (blooms) intenses (Fig. 3a). Certaines baisses étaient consécutives à des développements d'organismes filtreurs (daphnies et rotifères). Les développements excessifs (80 ind.1-1) de daphnies (Daphnia magna Straus) n'ont plus eu lieu après l'empoissonnement des enceintes expérimentales ( 0 ind.1-1), et les développements de rotifères (des genres Brachionus, Keratella et Epiphanes) sont restés limités après l'augmentation du temps de rétention $(<4000$ ind.1 1 alors qu'auparavant, des densités de 8000 à 9500 ind. $1^{-1}$ étaient fréquentes).

L'évolution des matières en suspension montre une nette évolution saisonnière puisque les biomasses algales récoltées dans chaque enceinte ont été de $2 \mathrm{~g} \mathrm{MES} \mathrm{sem}^{-1}$ (flux de $30 \mathrm{l}$ ) en hiver et de $140 \mathrm{~g}$ MES.sem-1 (flux de 200 l) en été. La valeur maxi-

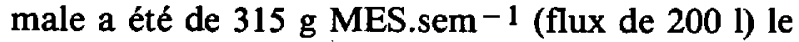
7 juillet 1992 dans la culture $\varphi_{2}$, ce qui est comparable aux productions de systèmes brassés. Cependant, des valeurs aussi élevées sont éphémères.

\subsection{Oxygène et pH}

Les cultures n'ont pas été saturées en oxygène avant l'hiver 1991/1992 (Fig. 4). A partir de cette époque, les milieux se sont progressivement oxygéné. Une sursaturation de $258 \%$ a même été observée le 18 février 1992. A partir du 28 avril, dans les deux milieux recevant une fertilisation minérale, les pourcentages de saturation ont été particulièrement élevés (près de $400 \%$ ), alors que la teneur en oxygène dissous dans les deux milieux fertilisés organiquement était nettement plus faible (jusqu'à $33 \%$ de déficit).

Le pH des cultures algales (Fig. 5) est resté relativement faible jusqu'en mars $1992(<8)$. A partir du mois d'avril, des pH élevés (9 voire 10 ) ont été constatés les 12 mai et 30 juin 1992, alors qu'une forte régression des populations algales était entrain de se produire.

\subsection{Eléments nutritifs minéraux}

- Azote

La concentration en azote ammoniacal a beaucoup varié (de $0 \mathrm{mg} .1^{-1}$ à $24,4 \mathrm{mg} . \mathrm{l}^{-1}$ ), en fonction de la fertilisation apportée (Fig. 6). Jusqu'au printemps 1992, la concentration moyenne a été très élevée (près de $8 \cdot \mathrm{mg} . \mathrm{l}^{-1}$ ), car la fertilisation n'était interrompue que lorsque la teneur en azote ammoniacal atteignait des seuils critiques ( $>15 \mathrm{mg} \cdot \mathrm{l}^{-1}$ ). Ces valeurs élevées n'étaient pas dues à un mauvais fonctionnement des cultures: l'élimination de l'azote ammoniacal a le plus souvent été très bonne, de l'ordre de $80 \%$.

L'épuration hebdomadaire moyenne a été de $52 \%$, mais l'écart-type a été très important pendant l'expérimentation. Ainsi, au cours de l'été 1991 et du printemps 1992, les cultures ont permis d'obtenir une excellente épuration (jusqu'à $100 \%$ ). En revanche, les performances au cours de l'automne et de l'hiver ont été beaucoup plus faibles ( $40 \%$ en moyenne). Une augmentation de la teneur en azote ammoniacal a même été obtenue sans qu'il y ait eu apport de lisier à l'issue de périodes de gel (10 décembre 1991, 28 janvier 1992).

A partir d'avril 1992, les apports d'engrais n'ont plus été effectués lorsque la concentration en $\mathrm{N}-\mathrm{NH}_{3}$ atteignait $5 \mathrm{mg} . \mathrm{l}^{-1}$ (Fig. 6b). Il en est résulté que près de $100 \%$ de l'azote ammoniacal introduit a été éliminé. Cette mobilisation paraît étroitement liée à la reprise de l'activité phytoplanctonique qui se traduit d'ailleurs par une augmentation du $\mathrm{pH}$ jusqu'à 9 voire 10 .

Les concentrations en nitrite et en nitrate sont restées faibles dans les cultures recevant du lisier $(<0,5$ mg.1 ${ }^{-1} \mathrm{~N}-\mathrm{NO}_{2} ;<4,5 \mathrm{mg} .1^{-1} \mathrm{~N}-\mathrm{NO}_{3}$ ), mais ont été beaucoup plus fortes avec une fertilisation à base d'ammonitrate (2 mg.1-1 $\mathrm{N}-\mathrm{NO}_{2} ; 25 \mathrm{mg} . \mathrm{l}^{-1} \mathrm{~N}$ $\mathrm{NO}_{3}$ ). Dans tous les milieux, l'élimination par les algues de ces nutriments a été faible et inférieure à $20 \%$.

\section{- Phosphate}

A la différence des éléments azotés; la concentration en phosphate a toujours été relativement stable et voisine de $3 \mathrm{mg} .1^{-1}$ (Fig. 7). Les variations observées sont directement liées aux apports de fertilisants. 

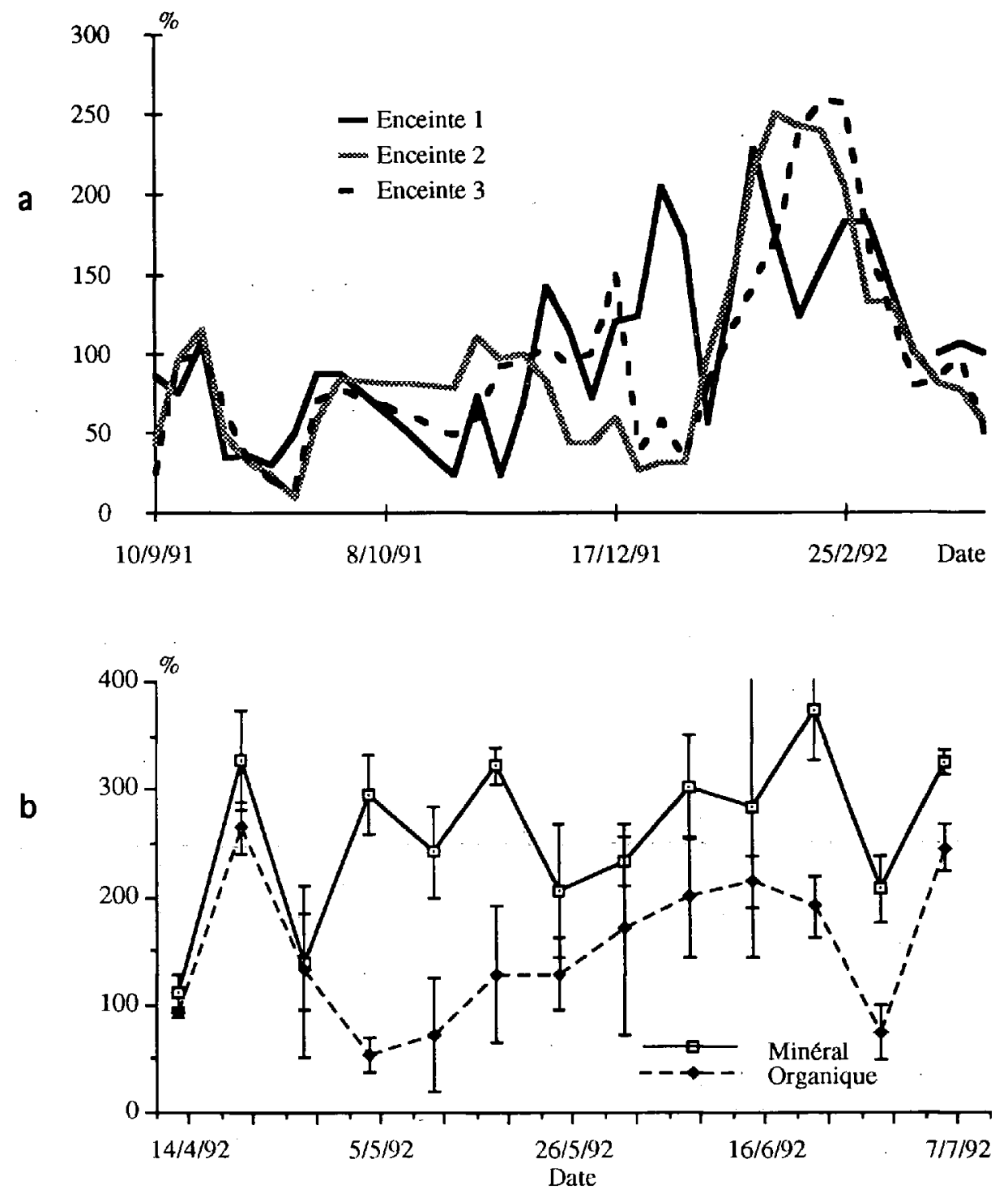

Fig. 4. Evolutions du pourcentage de saturation en oxygène dans trois enceintes de culture algale du 13 mai 1991 au 7 avril 1992 (a) et dans quatre enceintes (b) du 14 avril au 7 juillet 1992 (moyenne, et valeurs pour les deux enceintes qui reçoivent le fertilisant de même nature).

Fig. 4. Percentage of oxygen saturation in three tanks from May $13^{\text {th }} 1991$ to April $7^{\text {th }} 1992$ (a) and in four tanks (b) from April $14^{\text {th }}$ to July $7^{\text {th }} 1992$ (average and extreme values for two tanks receiving the same fertilizer). 

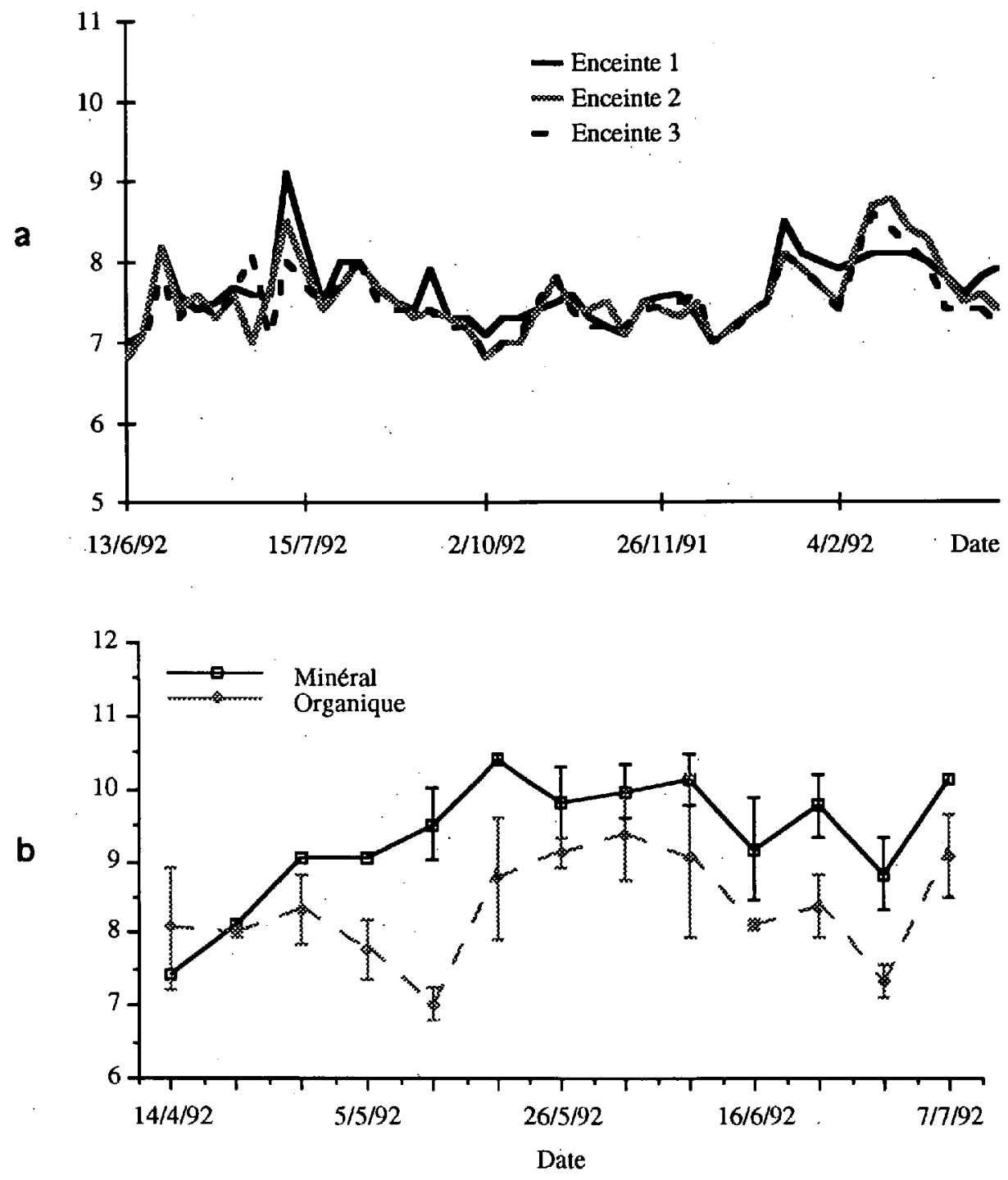

Fig. 5. Evolutions du pH dans trois enceintes de culture algale du 13 mai 1991 au 7 avril 1992 (a) et dans quatre enceintes (b) du 14 avril au 7 juillet 1992 (moyenne et valeurs pour les deux enceintes qui reçoivent le fertilisant de même nature).

Fig. 5. pH in three tanks from May 13 th 1991 to April $7^{\text {th }} 1992$ (a) and in four tanks (b) from April $14^{\text {th }}$ to July $7^{\text {th }} 1992$ (average and extreme values for two tanks receiving the same fertilizer). 

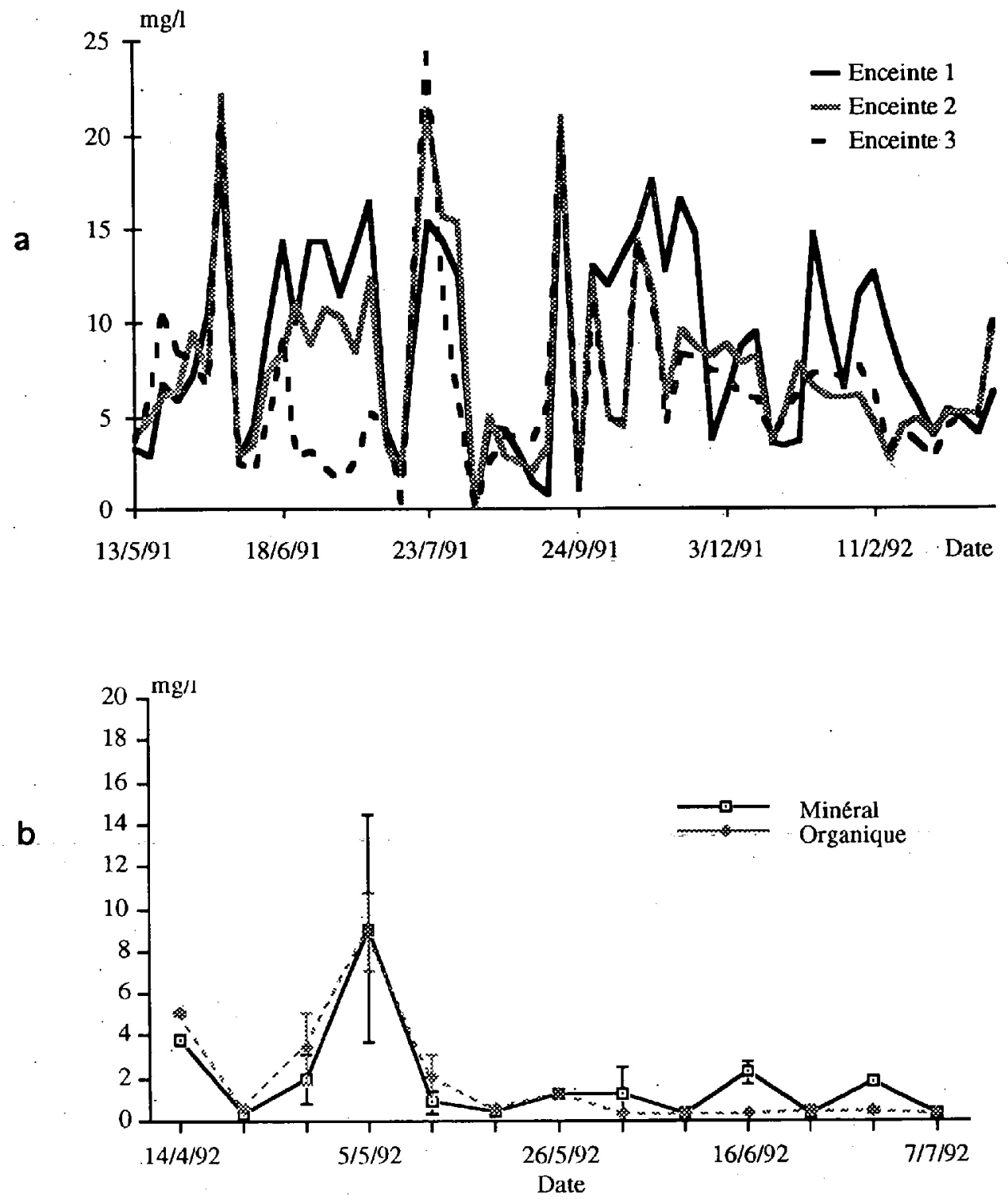

Fig. 6. Evolutions des concentrations en azote ammoniacal $\left(\mathrm{N}-\mathrm{NH}_{3}\right)$ dans trois enceintes de culture algale du 13 mai 1991 au 7 avril 1992 (a) et dans quatre enceintes (b) du 14 avril au 7 juillet 1992 (moyenne et valeurs pour les deux enceintes qui reçoivent le fertilisant de même nature).

Fig. 6. N-NH 3 concentrations in three tanks from May $13^{\text {th }} 1991$ to April $7^{\text {th }} 1992$ (a) and in four tanks (b) from April $14^{\text {th }}$ to July $7^{\text {th }} 1992$ (average and extreme values for two tanks receiving the same fertilizer). 

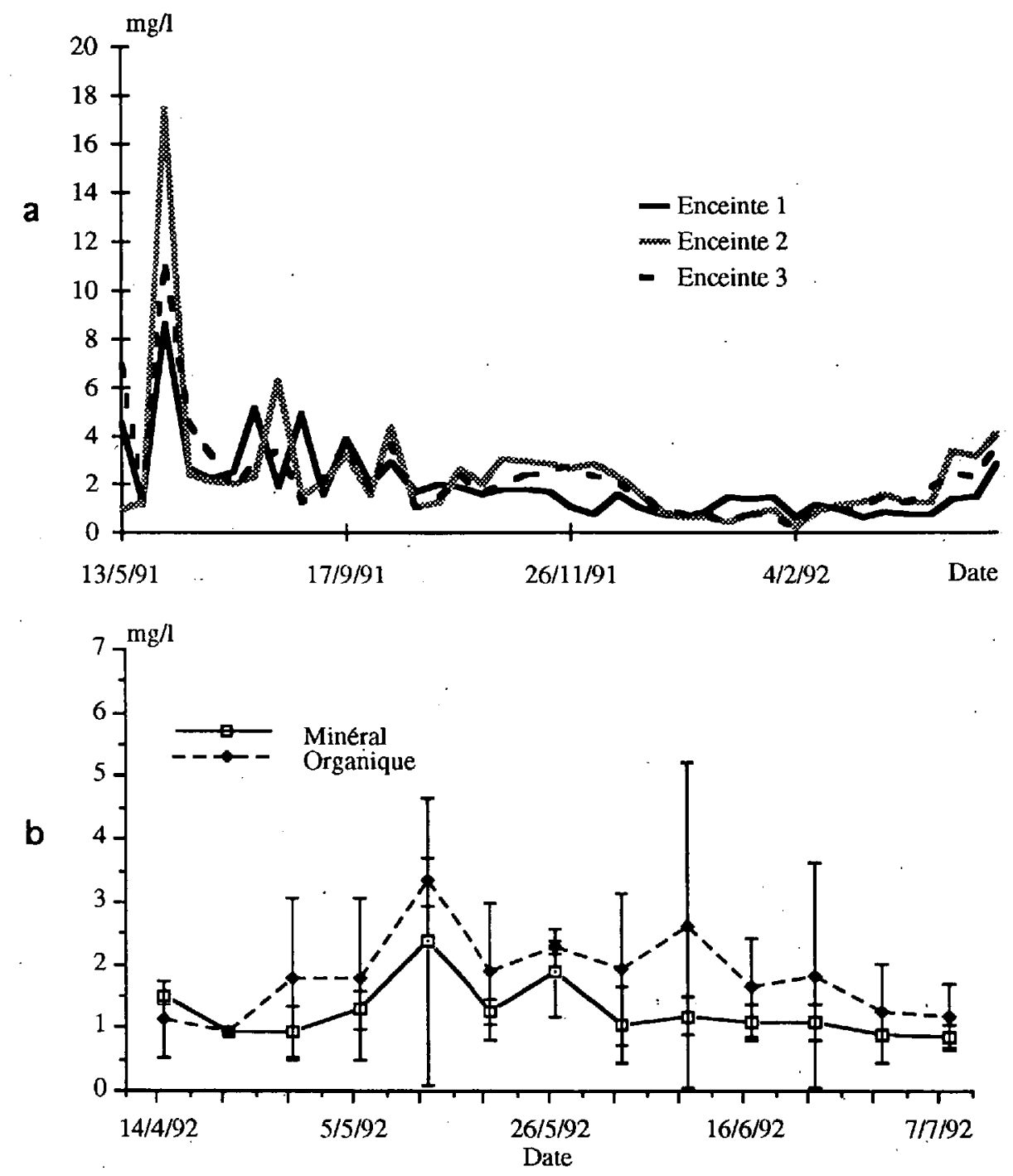

Fig. 7. Evolutions des concentrations en phosphate $\left(\mathrm{PO}_{4}{ }^{3-}\right.$ ) dans trois enceintes de culture algale du 13 mai 1991 au 7 avril 1992 (a) et dans quatre enceintes (b) du 14 avril au 7 juillet 1992 (moyenne et valeurs pour les deux enceintes qui reçoivent le fertilisant de même nature).

Fig. 7. $\mathrm{PO}_{4}{ }^{3-}$ concentrations in three tanks from May 13 th 1991 to April $7^{\text {th }} 1992$ (a) and in four tanks (b) from April 14 th to July $7^{\text {th }} 1992$ (average and extreme values for two tanks receiving the same fertilizer). 
Les pourcentages d'épuration sont de l'ordre de $50 \%$ en moyenne, mais ils sont plus faibles que pour l'azote ammoniacal. Ainsi, une épuration totale (100\%) n'a jamais été obtenue, ce qui prouve que la teneur en phosphate n'est pas un facteur limitant dans ces systèmes de culture algale.

\section{Discussion-Conclusion}

\subsection{Biomasse et activité algale}

\section{1:1. Biomasse}

Les espèces qui sont apparues sont caractéristiques des milieux riches en matières organiques et pollués (Angeli 1980). Plusieurs populations se sont succédées au cours du temps. Quand les algues des genres Chlamydomonas ou Cryptomonas se développent en masse, la fertilisation des cultures est excessive et il est impératif de l'interrompre pour éviter qu'une désoxygénation ne survienne et n'entraîne la mortalité de la culture (Dabbadie \& Gagnon, sous presse). Ces développements surviennent lorsque la concentration en $\mathrm{N}-\mathrm{NH}_{3}$ dépasse $10 \mathrm{mg} .1-1$. C'est pour éviter que cela ne se produise que, suite à cette constatation, les apports d'engrais n'ont plus été effectués lorsque la concentration en $\mathrm{N}-\mathrm{NH}_{3}$ atteignait $5 \mathrm{mg} \cdot \mathrm{l}^{-1}$.

La corrélation médiocre entre la concentration en chlorophylle a et le nombre total de cellules peut être imputable aux espèces présentes, à la taille des algues ou à leur état physiologique. La chlorophylle a est en effet plus abondante dans les cellules jeunes que pendant la phase de sénescence (Margalef 1963, Capblancs 1982). Il est également possible que l'environnement intervienne, puisque certaines espèces comme Chlorella s'adaptent à de nouvelles conditions d'éclairement, en modulant leur contenu en pigments (Bonin et al. 1981).

Les organismes du zooplancton (daphnies et rotifères principalement) qui apparaissent parfois dans les cultures sont susceptibles de provoquer une diminution rapide de la biomasse algale (Salomoni, sous presse, Combres et al., sous presse). S'il est facile d'éliminer les daphnies en empoissonnant les cultures avec des carpes communes, l'élimination des rotifères pose problème (Dabbadie \& Gagnon, sous presse). Ces organismes semblent en effet se développer lorsque les ressources trophiques de petite taille sont abondantes (bactéries, matières organiques, algues du nanoplancton, etc...), comme cela se produit lorsque la fertilisation organique est importante. Au cours de ces expérimentations, leurs développements ont pu être contrôlés lorsque le temps de rétention a été augmenté. Il se peut que les algues de grande taille (comme Scenedesmus sp. de $20 \mu \mathrm{m}$ ), qui se développent préférentiellement lorsque le temps de rétention est long, ne puissent pas entrer dans le régime alimentaire des rotifères. La taille des algues est en effet un facteur qui conditionne leur développement (Pourriot \& Champ 1982). Mais il se pourrait également que les populations algales denses aient un rôle dans ce contrôle. En effet, en présence d'un phytoplancton abondant, le rythme de filtration des rotifères augmente dans des proportions telles que les cellules ingérées traversent trop rapidement le tube digestif pour être assimilées. En outre, certaines algues excrètent ou libèrent lors de leur éclatement dans l'estomac des substances toxiques pour les rotifères (Lefèvre et al., 1952, Pourriot 1965).

\subsubsection{Activité algale}

L'oxygénation des cultures et leur $\mathrm{pH}$ résultent principalement de l'activité des algues et de celle des bactéries. Les algues, au cours de la photosynthèse produisent en effet de l'oxygène et provoquent une augmentation du pH en consommant le dioxyde de carbone. Pour les raisons inverses, les bactéries ont une action opposée.

Avant l'hiver 1991-1992, l'activité bactérienne était intense, en raison des apports massifs de lisier, ainsi qu'en attestent les faibles taux d'oxygénation et les bas pH. Cette activité a même provoqué la désoxygénation totale des cultures et la mort des algues (Dabbadie \& Gagnon, sous presse). Au cours de l'hiver, l'oxygénation s'est améliorée en raison des basses températures (aux basses températures, l'eau contient une plus grande quantité d'oxygène dissous et l'activité bactérienne est limitée) et grâce à la réduction des apports de lisier. Néanmoins, l'activité algale est restée faible et n'a pas permis une augmentation significative du $\mathrm{pH}$. En revanche, au printemps les augmentations de la teneur en oxygène et du pH attestent de la reprise d'activité du phytoplancton. Mais, les différences observées entre les milieux recevant du lisier et ceux recevant une fertilisation minérale doivent être attribuées à l'activité bactérienne. Les fertilisants organiques favorisent en effet les développements de bactéries (Schroeder 1978).

Les faibles activités algales pendant l'hiver ne sont pas surprenantes, compte tenu des basses températures 
et de la faible luminosité qui dominent en cette saison. Cependant, dans le cas présent où les cultures algales sont réalisées dans un but d'épurer des effluents organiques comme le lisier (qui sont produits en toute saison), cette contrainte pose un problème technique de gestion des déchets pendant l'hiver.

Toutefois, il semblerait que les algues qui se développent dans ces cultures soient, pendant l'hiver, beaucoup plus limitées par une basse température que par une luminosité faible (Dabbadie \& Gagnon, sous presse). Ceci est d'ailleurs confirmé par des expériences réalisées au Muséum où une élévation de la température jusqu'à $1.0^{\circ} \mathrm{C}$ a permis d'obtenir plus de $800 \mu \mathrm{g} .1^{-1}$ de chlorophylle a au mois de février 1990 (Sevrin-Reyssac, non publié). Les espèces algales qui dominent dans ces milieux ont en effet un mode de nutrition hétérotrophe qui leur permet de se développer en l'absence de lumière, en utilisant directement certains composés organiques.

Dans ces conditions, la mise en place de systèmes de chauffage ou de limitation des pertes de chaleur (tels que des serres) pourrait se révéler rentable en augmentant de façon significative l'efficacité hivernale des dispositifs d'épuration biologique faisant appel aux cultures d'algues. Il faut cependant que ces dispositifs ne nécessitent pas une trop grande technicité, ni une trop grande surface, en d'autres termes, qu'ils soient rustiques sans être trop onéreux. Des expérimentations devront être menées pour vérifier que de telles installations restent, malgré le coût supplémentaire, plus avantageuses que les autres technologies d'épuration des lisiers.

\subsection{Sels nutritifs}

Les sels nutritifs abondants dans le lisier et responsables des problèmes de pollution par ce déchet sont très bien épurés par les microalgues. L'azote ammoniacal et le phosphate sont en effet les formes d'azote et de phosphore qui sont utilisées préférentiellement par le phytoplancton (Lepailleur 1988, Capblanc 1982). Cependant, à pH élevé comme cela fut le cas au printemps 1992, une fraction importante de l'azote ammoniacal est sous forme gazeuse (à pH 10 et à $20^{\circ} \mathrm{C}, 79,9 \%$ de l'azote ammoniacal est sous forme de $\mathrm{NH}_{3}$ ). Il est donc possible, lorsque les conditions sont très favorables à l'activité du phytoplancton, qu'une part non négligeable de l'azote diffuse par « stripping » dans l'atmosphère.
Bien que l'épuration du nitrite et du nitrate soit beaucoup plus faible, ces éléments ne posent pas de problème de pollution car leurs concentrations sont peu importantes. De plus, il paraît vraisemblable que leurs fluctuations ne soient pas dues aux prélèvements des algues (qui semblent mineurs dans ces milieux riches en azote ammoniacal), mais à des réactions de dénitrification qui aboutissent finalement à la production de $\mathrm{N}-\mathrm{NH}_{3}$, qui est une forme bien épurée.

Tous ces résultats ne doivent pas être extrapolés à la station pilote de Château-Thierry, en raison du changement d'échelle. Néanmoins, grâce aux connaissances acquises lors des expérimentations réalisées au Muséum, les premiers résultats obtenus paraissent très prometteurs : une concentration de $1.6 \mathrm{mg} .1^{-1}$ de chlorophylle a a été obtenue le 17 août 1992, dans les premières semaines de fonctionnement, et aucune nuisance n'a pu être observée (Sevrin-Reyssac, non publié).

\section{Remerciements}

Ce travail n'aurait pu être réalisé sans l'appui constant de Mme J. Sevrin-Reyssac du Muséum d'Histoire Naturelle, ni sans le soutien financier du Ministère de la Recherche et de la Technologie, du Ministère de l'Agriculture et de la Forêt, et des échanges interuniversitaires France-Québec (Université Laval).

\section{Travaux cités}

Amoros C. 1984. - Introduction pratique à la systématique des organismes des eaux continentales françaises. Crustacés cladocères. Bull. Soc. linn. Lyon, 3,4 : 1-63.

Angeli N. 1980. - Interactions entre la qualité de l'eau et les éléments de son plancton. In : La pollution des eaux continentales. Incidence sur les biocénoses aquatiques. P. Pesson (ed), Gauthier-Villars, Paris. 345 p : 97-146.

Bonin D.J., Maestrini S.Y. \& Leftley J.W. 1981. - Some processes and physical factors that effect the ability of individual species of algae to compete for nutrient partition. In : Physiological bases of phytoplankton ecology. T. Platt (ed), Can. Bull. Fish. Aquat. Sci. 210, Ottawa. 346 p : 292-309.

Bourrelly P. 1981. - Les algues d'eau douce, T. II : Algues jaunes et brunes. Soc. Nouv. Ed. Boubée, Paris. 517 p.

Bourrelly P. 1985. - Les algues d'eau douce, T. III : Algues bleues et rouges. Soc. Nouv. Ed. Boubée, Paris. 606 p.

Bourrelly P. 1990 . - Les algues d'eau douce, T. I : Algues vertes. Soc. Nouv. Ed. Boubée, Paris. 572 p.

Capblancq J. 1982. - Phytoplancton et production primaire. In : Ecologie du plancton des eaux continentales. R. Pourriot, J. Capblancq, P. Champ et J.A. Meyer (eds), Masson, collection d'écologie 16, Paris, 198 p : 1-48.

Cardinal C. 1979. - Algues planctoniques du bassin de la Seine (à l'exception des cyanophycées et des diatomées). Bull. Mus. natn. Hist. nat. Paris, $4^{e}$ sér., section B, $1(1,4): 285-327$. 
Combres C., Sevrin-Reyssac J. \& Djonga J.L. sous presse. Elevages de daphnies intégrés à un système de recyclage biologique du lisier de porc In : Actes colloq. aquac. (Bordeaux, mars 1992). Europ. Aquac. Soc., Bredene.

Dabbadie L. \& Gagnon N. sous presse. - Nuisances rencontrées en élevages intensifs de microalgues recevant du lisier de porc. In : Actes colloq. aquac. (Bordeaux, mars 1992). Europ. Aquac. Soc., Bredene.

De la Nouë J. \& Proulx D. 1986 . - Intérêt des biomasses d'algues et d'invertébrés obtenues par recyclage. Eutropie, 130, $131: 17-32$.

De la Nouë J., Proulx D., Dion P. \& Gudin C. 1990 . - Drugs and chemicals from aquaculture. In : Aquaculture Europe 89, N. de Pauw \& R. Billard (eds), EAS spec. public. 12, Bredene, $465 \mathrm{p}: 389-418$.

De Pauw N., de Leenheer Jr. L., Laureys P., Morales J. \& Reartes J. 1980. - Cultures d'algues et d'invertébrés sur déchets agricoles. In : La Pisciculture en étang. R. Billard (ed), INRA publ., Paris. 434 p : 189-214.

Dussart B. 1966. - Limnologie. L'étude des eaux continentales. Gauthier-Villars, Collect. géobiol., écol., aménag., Paris. $677 \mathrm{p}$.

HACH 1991. - DR/2000 spectrophotometer handbook, Hach company, Loveland, Colorado. 559 p.

Lefèvre M., Jakob H. \& Nisbet M. 1952. - Auto et hétéroantagonisme chez les algues d'eau douce in vitro et dans les collections d'eau naturelle. Ann. Stat. Centr. Hydrobiol. appl., $4: 5-198$.
Lepailleur H. 1988. - Les algues d'eau douce. In : Biologie des eatux. Moyens et techniques. D. Champiat \& J.P. Larpent (eds), Masson, Paris. $374 \mathrm{p}: 34-42$.

Margalef R. 1963. - Modelos simplificados del ambiente marino para el estudio de la sucesiòn y distributiòn del fitoplancton y del valor indicator de sus pigmentos. Invest. pesq., 23: $11-52$.

Pourriot R. 1965. - Recherches sur l'écologie des Rotifères. Thèse doct. Fac. sci. Univ. Paris. 224 p.

Pourriot R. \& Champ P. 1982 . - Consommateurs et production secondaire. In : Ecologie du plancton des equx continentales. R. Pourriot, J. Capblancq, P. Champ \& J.A. Meyer (eds), Masson, collection d'écologie 16, Paris. 198 p : 49-112.

Pourriot R. \& Francez A.J. 1986. - Introduction pratique à la systématique des organismes des eaux continentales françaises ; Rotifères. Bull. Soc. linn. Lyon. 8 : 1-37.

Salomoni $C$., sous presse. - La production de matière vivante par recyclage d'effluents d'élevage y compris de pisciculture dans les systèmes de lagunage. In : $L$ 'aquaculture des cyprinidés (réed). R. Billard (ed), INRA publ., Paris.

Schroeder G.L. 1978. - Autotrophic and heterotrophic production of microorganisms in intensively manured fish ponds, and related fish yields. Aquaculture, $14: 303-325$.

Utermöhl H. 1958. - Zur Vervollkommung der quantitativen Phytoplankton-Methodic. Mitt. int. Verein theor. angew. Limnol., $9: 38 \mathrm{p}$.

Ward H.B. \& Whipple G.C. 1918. - Fresh-water biology. John Wiley \& sons Inc, New-York. 1111 p. 